05

\title{
О возможном механизме внешнего инфразвукового механического стимулирования процесса формирования нанокристаллов в аморфной металлической пленке
}

\author{
(C) Е.Е. Слядников, ${ }^{1,2,3}$ И.Ю. Турчановский ${ }^{2}$ \\ ${ }^{1}$ Институт фризики прочности и материаловедения СО РАН, \\ 634055 Томск, Россия \\ ${ }^{2}$ Федеральный исследовательский центр Институт вычислительных технологий СО РАН, \\ 630090 Новосибирск, Россия \\ ${ }^{3}$ Томский университет систем управления и радиоэлектроники, \\ 634050 Томск, Россия \\ e-mail: eeslyadnikov@gmail.com
}

Поступило в Редакцию 13 февраля 2021 г.

В окончательной редакции 24 апреля 2021 г.

Принято к публикации 28 мая 2021 г.

\begin{abstract}
Сформулированы кинетическая модель, физическая причина и условие стимулированного внешними инфразвуковыми механическими колебаниями процесса формирования нанокристаллов в аморфной металлической пленке. За эти процессы ответственны наноструктурные элементы аморфной среды: локально упорядоченные нанокластеры и нанообласти, содержащие свободный объем, в которых содержатся двухуровневые системы. При деформации стекла происходит возбуждение двухуровневых систем, благодаря чему они дают существенный вклад в неупругую деформацию, структурную релаксацию, образование нанокластеров и нанокристалов. Физический механизм нанокристаллизации металлического стекла при механическом воздействии включает в себя помимо механизма локальных термических флуктуаций также атермический механизм квантового туннелирования атомов или атомных групп, стимулированный неупругой деформацией. По этому механизму упорядоченный нанокластер перестраивается в нанокристалл, возникает нанометровая потенциальная яма, в которой локализуется электрон проводимости с образованием фазона. Критический радиус фазона равен $\sim 0.5 \mathrm{~nm}$, а критическое значение глубины нанометровой потенциальной ямы (условие, при котором образуется стабильный фазон) составляет приблизительно $1 \mathrm{eV}$.
\end{abstract}

Ключевые слова: кинетическая модель, нанокристаллы, аморфная металлическая пленка, инфразвук, неупругая деформация, квантовое туннелирование, релаксация, локализация электрона проводимости, фазон.

DOI: $10.21883 /$ JTF.2021.11.51526.35-21

\section{Введение}

Аморфные металлические сплавы нашли широкое применение в электротехнике, радио и космической технике [1]. Уникальными механическими свойствами обладают аморфные металлические сплавы с наноструктурой, которая имеет вид беспорядочно ориентированных нанокристаллов в аморфной матрице [2,3]. Для наноструктурирования закаленной из жидкого состояния аморфной пленки используются низкочастотные механические колебания при температуре намного ниже температуры стеклования, причем деформация образца остается в пределах упругой области [4]. Образцы аморфной тонкой ленты на основе сплава TiNi толщиной $40 \mu \mathrm{m}$, шириной $1.6 \mathrm{~mm}$ и длиной $10 \mu \mathrm{m}$ подвергали десятиминутному воздействию инфразвука с частотой $20 \mathrm{~Hz}$ и амплитудой смещения $1 \mu \mathrm{m}$ и $4 \mu \mathrm{m}$ при температуре $T_{0}=25^{\circ} \mathrm{C}$. До и после низкочастотной обработки структура аморфного сплава была исследована методами рентгеновской дифракции (XRD) и просвечивающей электронной микроскопии высокого разрешения (HRTEM). Данные XRD и HRTEM свидетельствуют о том, что исходный сплав полностью аморфный; структура образца, подвергнутого механическим колебаниям с амплитудой $1 \mu \mathrm{m}$, близка к структуре изначально необработанного образца (изменяется ближний порядок и уменьшается свободный объем); в структуре образца, подвергнутого механическим колебаниям с амплитудой $4 \mu \mathrm{m}$, в аморфной матрице возникают несферические кластеры с правильными положениями атомов и размерами 3-5 nm.

Установлено [4], что при механическом воздействии скрытая теплота кристаллизации немного уменьшается, т.е. свободная энергия состояния с нанокристаллами немного понижается по сравнению со свободной энергией аморфного состояния. Химический и фазовый составы сплавов с нанокристаллами и без них одинаковые, поэтому причина инфразвукового стимулирования нанокристаллизации не связана с диффузией и вызвана коллективными атомными перегруппировками при структурной релаксации.

Дальнейшие экспериментальные исследования показали [5], что увеличение продолжительности колебаний до $2 \mathrm{~h}$ увеличивает размер кластеров, причем их рост 
может быть разным в разных направлениях. После механических колебаний в течение $4 \mathrm{~h}$ пленка приобретает кристаллическую структуру, состоящую из зерен неправильной формы и различных кристаллографических ориентаций, в которые вкраплены аморфные островки.

Механизм такого влияния механических колебаний на нанокристаллизацию аморфного материала остается неясным.

Согласно классическим представлениям [2,3,6], зарождение кристаллов в аморфных сплавах может происходить по гомогенному или гетерогенному механизму. В окрестности температуры стеклования гомогенное зарождение происходит путем флуктуационного (спонтанного) образования зародыша с радиусом, большим критического $[2,3,6]$. Ниже температуры стеклования для процесса кристаллизации существенную роль играет гетерогенное зародышеобразование (на дефектах, границах и т. д.) $[2,3,6,7]$ и зародышеобразование, обусловленное „вмороженными“ центрами кристаллизации $[2,3,8]$.

Однако типичные плотности гетерогенных зародышей в металлических расплавах составляют $10^{11}-10^{12} \mathrm{~m}^{-3}$. Даже в аморфных сплавах, в которых нет наноразмерных кристаллитов, плотность кристаллитов заметно превышает эти значения (например, в сплаве $\mathrm{Fe}_{80} \mathrm{~B}_{20}$ плотность кристаллитов составляет $10^{18} \mathrm{~m}^{-3}$ [9]). В наноструктурных системах (например, в сплавах $\mathrm{Cu}-\mathrm{Ti}[10]$ ) плотность кристаллитов может достигать $10^{25} \mathrm{~m}^{-3}$, поэтому большая часть зародышей, вероятнее всего, возникает путем гомогенного зарождения в процессе отжига.

Анализ физических условий, в которых протекает нанокристаллизация стационарным отжигом и механическим воздействием [2-5], показывает, что механизмы нанокристаллизации аморфной пленки при этих воздействиях существенно различаются. При термическом отжиге температура образца значительно выше комнатной, процесс нанокристаллизации протекает медленно и в основном гомогенно, а нанокристаллы возникают по всему объему термофлуктуационным путем.

При механическом воздействии температура среды комнатная, поэтому вероятность образования нанокристалла обычным термофлуктуационным путем много меньше, чем при температуре отжига, вследствие большой энергии активации процесса образования нанокристалла. Однако при механических колебаниях с амплитудой $4 \mu \mathrm{m}$ в пленке возникают значительные сдвиговые напряжения. При критическом значении сдвигового напряжения среда теряет сдвиговую устойчивость, возникает неупругая необратимая деформация. Поэтому механизм нанокристаллизации металлического стекла при механическом воздействии должен включать в себя, помимо механизма локальных термических флуктуаций, также атермический механизм квантового туннелирования атомов или атомных групп [11-13], стимулированный неупругой необратимой деформацией. Способность аморфных металлических сплавов к неупругой деформации, структурной релаксации и образованию нанокристалов связана с коллективизированным металлическим ха- рактером межатомной связи, при которой гораздо легче могут быть осуществлены процессы коллективных атомных перемещений [2,3]. Поэтому при построении механизма неупругой деформации, структурной релаксации и нанокристаллизации металлического стекла необходимо учитывать влияние электронной подсистемы [14-16], а конкретно для металлического стекла - локализацию электрона проводимости в нанометровой потенциальной яме и образование фазона [16].

Целью настоящей работы является формулировка возможного физического механизма, кинетической модели, физической причины и условия стимулированного инфразвуком неравновесного перехода первого рода в металлической пленке из аморфного состояния в наноструктуру. Этот подход должен учитывать как термический, так и атермический механизмы неупругой деформации, структурной релаксации, нанокристаллизации, а также локализацию электрона проводимости в потенциальной яме возникшего нанокристалла и образование фазона.

\section{1. Физический механизм неупругой деформации металлических стекол}

Неупругая деформация объясняется локальными статическими смещениями атома или группы атомов из начальных положений равновесия в новые положения равновесия, расположенные от начальных положений на расстоянии меньше межатомного в ответ на приложенное напряжение сдвига $\sigma$ [17-21]. Вероятность такой атомной перестройки за счет тепловой флуктуации пропорциональна $\exp \left(-\frac{\left(E_{a}-\sigma a_{0}^{3}\right)}{k T}\right)$, где $E_{a}-$ энергетический активационный барьер перестройки, $a_{0}-$ межатомное расстояние. В области пластической деформации считается, что $\left(E_{a}-\sigma a_{0}^{3}\right) \leq 0$, а при неупругой деформации - $\left(E_{a}-\sigma a_{0}^{3}\right)>0$, следовательно, неупругая деформация является термоактивируемой. Однако эффект неупругой деформации экспериментально наблюдается при криогенной температуре $(77 \mathrm{~K})[22]$, где энергия тепловой флуктуации невелика $(0.007 \mathrm{eV})$, и при более низких температурах [23]. Поэтому актуальным является установление физического механизма неупругой деформации металлических стекол в области комнатных температур и ниже.

Первая микроскопическая модель элементарных кооперативных неупругих перестроек в металлических стеклах, построенная на концепции однородно распределенного свободного объема, предложена Аргоном [17]. В модели [18] предполагается, что вследствие неоднородности структуры стекла в нем возникают области с избыточным относительно „идеальной структуры“ свободным объемом - „центры релаксации“. В настоящее время часто используемыми являются модель спектра энергий активации [19], а также модель направленной структурной релаксации, ориентированной внешней силой [20]. В модели [21] предложен метод описания 
локальной структуры аморфных сплавов, основанный на концепции $n$-, $p$ - и $\tau$-дефектов.

В работе [24] предложена поликластерная модель структуры аморфного состояния, в которой элементарные перестройки атомных конфигураций под действием внешних сил происходят на межкластерных границах, причем при высоких температурах - по механизму тепловых флуктуаций, а при низких температурах по механизму квантового туннелирования. Стоит также упомянуть о более ранних работах $[25,26]$, которые оказали существенное влияние на современные представления о механизме неупругой деформации в твердых телах. Показано [25], что если атомы примеси обладают высокой подвижностью, как, например, водород в металлах, то возникает диффузионная релаксация в условиях градиента деформаций. В [26] предложен недиффузионный механизм мартенситного фазового превращения, согласно которому элементарная ячейка новой фазы получается в результате спонтанного искажения (неупругой перестройки) ячейки старой фазы.

Сопоставляя поликластерную модель [24] и модель свободного объема [17-21], заметим, что в последней неупругие перестройки атомов под действием напряжения происходят в местах локализации вакантных полостей, причем наличие таковых и их локальная структура не связываются с существованием локального упорядочения. Поэтому независимо от природы, происхождения и распределения свободного объема его роль в осуществлении процессов неупругой деформации, структурной релаксации в закаленных из жидкого состояния металлических стеклах является определяющей.

Механические свойства металлических стекол являются структурно чувствительными, поэтому зависят от способа приготовления аморфного состояния [2-5]. При „закалке“ из жидкого состояния появление дислокаций, дисклинаций и других дефектов в системе маловероятно, поэтому для построения механизма неупругой деформации, структурной релаксации в закаленном металлическом стекле наиболее адекватно применение модели свободного (избыточного) объема [17-21] и поликластерной модели [24].

Особый интерес представляют структурные элементы аморфной среды („центры релаксации“), содержащие свободный объем и способные перестраиваться не только в результате тепловых флуктуаций, но и благодаря квантовому туннелированию. Атом, движущийся в двухъямном потенциале, создаваемом окружающими его атомами, или группа атомов, которая может образовывать две или более конфигураций с мало отличающимися энергиями при практически неизменном положении всех остальных атомов структуры, представляет собой двухуровневую систему [11-13,24]. Переход атома из одной ямы в другую или переход одной конфигурации атомов в другую, означающий переход с одного энергетического уровня на другой, при комнатных и более низких температурах может происходить благодаря квантовому туннелированию. Это имеет место, когда двухуровневая система близка к вырожденной, т.е. уровни нулевых колебаний атома в каждой из соседних ям без учета туннелирования должны отличаться мало. Начальная расстройка резонанса $\Delta_{0} \ll 1 \mathrm{eV}$, а начальная частота туннельных переходов $\omega_{K}^{0}=\omega_{0} \exp \left(-\frac{a}{a_{d B}}\right)$, с одной стороны, должна быть много меньше $\Delta_{0}$, чтобы начальное расщепление уровней $E_{0}=\sqrt{\Delta_{0}^{2}+\hbar^{2}\left(\omega_{K}^{2}\right)^{2}}$ было малым $(\sim \Delta)$, а с другой стороны, не слишком малой, чтобы туннелирование могло проявиться за время проведения эксперимента. Здесь $a_{d B}$ - длина волны де Бройля, $a$ - ширина потенциального барьера в двухъямном потенциале, $\omega_{0} \approx 10^{13} \mathrm{~Hz}$.

Для оценки характерных параметров туннелирования атома проведем модельный расчет. Пусть в нанообласти, обладающей свободным объемом, атом находится в двухъямном потенциале или существует группа атомов, способных образовать две конфигурации с мало отличающимися энергиями [11-13,24]. Потенциальную энергию системы можно представить в виде суммы двух одноямных потенциалов $U_{L}$ и $U_{R}$. В начальный момент времени при отсутствии механической нагрузки левая $(L)$ и правая $(R)$ потенциальные ямы имеют разную глубину, левая яма глубже правой. Действие на систему внешней механической силы приводит к изменению расстояний между атомами среды (деформации), что, в свою очередь, изменяет форму двухъямного потенциала.

Пусть известны волновые функции $\Psi_{L}, \Psi_{R}$ и собственные значения энергии $E_{L}, E_{R}$ для уравнения Шредингера с одноямными потенциалами $U_{L}, U_{R}$. Полный гамильтониан одномерной системы с двухъямным потенциалом $U_{L}+U_{R}$ имеет вид [27]:

$$
\begin{gathered}
H=-\frac{\hbar^{2}}{2 m} \frac{\partial^{2}}{\partial x^{2}}+U_{L}+U_{R}, \\
\Psi=a_{L} \Psi_{L}+a_{R} \Psi_{R}, \quad \Delta=E_{L}-E_{R} .
\end{gathered}
$$

Предполагая слабое перекрывание волновых функций $\Psi_{L}$ и $\Psi_{R}\left(S=\int d x \Psi_{L} \Psi_{R} \ll 1\right)$, из (1) находим энергию стационарных уровней $E_{ \pm}$

$$
\begin{gathered}
E_{ \pm}=\frac{1}{2}\left[E_{L}+E_{R} \pm \sqrt{D}\right], \quad D=\Delta^{2}+4 U_{L R}^{L} U_{L R}^{R}, \\
U_{L R}^{L}=\int d x \Psi_{L} U_{L} \Psi_{R}
\end{gathered}
$$

и стационарные состояния $\Psi_{ \pm}$, в которых атом делокализован между двумя ямами. Линейная комбинация стационарных волновых функций $\Psi(x, t)$, удовлетворяющая временно́му уравнению Шредингера с гамильтонианом (1) и начальному условию $\Psi(t=0)=\Psi_{L}$, позволяет найти вероятность нахождения атома в левой яме

$$
\begin{gathered}
W(t)=a(t)^{2}=1-\frac{16(\Delta+\sqrt{D})^{2} U_{L R}^{L} U_{L R}^{R}}{\left[(\Delta+\sqrt{D})^{2}+4 U_{L R}^{L} U_{L R}^{R}\right]^{2}} \sin ^{2}\left(\frac{\sqrt{D}}{2 \hbar} t\right) \\
a(t)=\int d x \Psi(x, t) \Psi_{L}(x) .
\end{gathered}
$$


$W(t)$ является периодической функцией времени, осциллирует от единицы до некоторого минимального значения, которое определяется расстройкой резонанса $\Delta$ и величиной произведения $U_{L R}^{L} U_{L R}^{R}$.

Если $\Delta=0$, то

$$
W(t)=1-\sin ^{2}\left(\frac{\sqrt{U_{L R}^{L} U_{L R}^{R}}}{\hbar} t\right) .
$$

Вероятность найти атом в левой яме меняется от единицы до нуля с периодом

$$
\tau=\frac{\pi \hbar}{\sqrt{U_{L R}^{L} U_{L R}^{R}}}
$$

и атом одинаковое время проводит в обеих ямах. Если $\Delta^{2} \gg 4 U_{L R}^{L} U_{L R}^{R}$, то

$$
W(t)=1-\frac{4 U_{L R}^{L} U_{L R}^{R}}{\Delta^{2}} \sin ^{2}\left(\frac{\Delta}{2 \hbar} t\right) .
$$

Атом практически все время находится в левой потенциальной яме. Когда внешняя механическая сила равна нулю, реализуется случай $\Delta<0, \Delta^{2} \gg 4 U_{L R}^{L} U_{L R}^{R}$ и атом находится только в левой яме. При критическом значении деформации достигается случай $\Delta=0$ и атом туннелирует в правую яму. Дальнейшее увеличение деформации приводит к случаю $\Delta>0, \Delta^{2} \gg 4 U_{L R}^{L} U_{L R}^{R}$, и атом находится только в правой яме. Поскольку неупругое смещение атома является элементарным актом структурной релаксации среды под нагрузкой и сопровождается релаксацией напряжений, это приводит к изменению положений атомов из ближайшего окружения и ,замораживанию“ измененной формы двухъямного потенциала. В результате чего атом не возвращается в левую яму, а остается в правой яме после снятия нагрузки. Так протекает элементарная неупругая деформация в металлическом стекле при $T=0$.

Расщепление энергетических уровней, соответствующее двум атомным конфигурациям, между которыми совершаются переходы, выражается через $\Delta$ и $\hbar \omega_{K}=\sqrt{4 U_{L R}^{L} U_{L R}^{R}}$, следующим соотношением: $E=$ $=\sqrt{\hbar^{2} \omega_{K}^{2}+\Delta^{2}}$. В стекле имеется большое число таких двухуровневых систем (центров релаксации), которые характеризуются разницей энергий (расстройкой резонанса) $\Delta$ в двух состояниях и частотой туннельного перехода $\omega_{K}=\omega_{0} \exp \left(-\frac{a}{a_{d B}}\right)$. Значения обеих этих величин статистически распределены в некоторых интервалах. Исследование распределения параметров двухъямных потенциалов указывает на возможность существования двух групп таких потенциалов - мягких (с междуямным барьером $V \sim 10^{-4}-10^{-3} \mathrm{eV}$ ) и жестких $\left(V \sim 10^{-1}-1 \mathrm{eV}\right)[11-13,24]$. В области температур $T \approx 1-300 \mathrm{~K}$ активируются тепловые перескоки между состояниями, величина барьера которых равна $V \leq 10^{-4}-2.4 \cdot 10^{-2} \mathrm{eV}$, поэтому для двухуровневой системы с жестким потенциалом механизм квантового туннелирования более вероятен.
В рамках одночастичной модели взаимодействия фононов с двухуровневыми системами [11-13,24] затравочная разность энергий $\Delta$, которую также называют параметром асимметрии, перенормируется, и расстояние между двумя уровнями двухуровневой системы становится равным $E=\sqrt{\hbar^{2} \omega_{K}^{2}+\Delta^{2}}$. В реальных системах возможны переходы между этими диагональными состояниями. Они обусловлены связью двухуровневой системы с колебаниями атомов внешнего окружения и сопровождаются испусканием или поглощением фононов. При прохождении фононной волны происходит смещение атомов, формирующих потенциальную кривую двухуровневой системы. При этом искажаются отдельные ямы кривой, что приводит к смещению затравочных уровней $\delta \Delta$. Также изменяется форма потенциального барьера, что приводит к изменению затравочной частоты туннельных переходов $\delta \omega_{K}$ :

$$
\delta \Delta=B_{i j} \varepsilon_{i j}, \delta \omega_{K}=D_{i j} \varepsilon_{i j}, \quad \varepsilon_{i j}=\frac{1}{2}\left(\frac{\partial u_{i}}{\partial x_{j}}-\frac{\partial u_{j}}{\partial x_{i}}\right) .
$$

Здесь $B_{i j}, D_{i j}$ - тензоры потенциалов деформации, которые зависят от локальной структуры стекла в том месте, где расположена двухуровневая система, $\varepsilon_{i j}-$ тензор деформации, который выражается через компоненты вектора смещений $u_{i}$. Приходящаяся на один атом энергия за один цикл деформации $\varepsilon=4 \cdot 10^{-4}$ равна $e_{a} \approx 1 \cdot 10^{-6} \mathrm{eV}$, поэтому $\delta \Delta \approx e_{a} \approx 1 \cdot 10^{-6} \mathrm{eV}$. Поскольку $\Delta_{0}<0$, можно оценить $\left|\Delta_{0}\right| \approx \delta \Delta \approx 1 \cdot 10^{-6} \mathrm{eV}$ и максимальную величину $\omega_{K} \approx 10^{9} \mathrm{~s}^{-1}$.

При деформации стекла происходит возбуждение двухуровневых систем, благодаря чему они дают существенный вклад в неупругую деформацию, структурную релаксацию, образование нанокластеров и нанокристалов. Из (4) видно, что морфология (форма) образующихся при неупругой деформации нанокластеров и нанокристаллов является анизотропной и определяется тензором потенциалов деформации, которые зависят от локальной структуры стекла в том месте, где расположена двухуровневая система.

Исследуем туннельный переход атома из одной потенциальной ямы в другую при конечной температуре с помощью формализма оператора плотности $\rho$ [28]. В этом случае атом, совершающий переходы между ямами, взаимодействует с термостатом (твердофазным окружением, которое при переходах сохраняет температуру). Используя в качестве базисных векторов собственные векторы $\Psi_{L}, \Psi_{R}$, с точностью до степени $S$ получим дифференциальные уравнения для матричных элементов оператора плотности $\rho$

$$
\begin{gathered}
i \hbar \frac{\partial \rho_{L L}}{\partial t}=U\left(\rho_{R L}-\rho_{L R}\right)-\frac{i \hbar}{\tau_{L L}}\left(\rho_{L L}-\rho_{L L}^{0}\right), \\
U \equiv U_{L R}^{L}=U_{L R}^{R}, \quad \tau_{i j}=\tau_{j i}, \quad i, j=L, R, \\
i \hbar \frac{\partial \rho_{R R}}{\partial t}=U\left(\rho_{L R}-\rho_{R L}\right)-\frac{i \hbar}{\tau_{L L}}\left(\rho_{R R}-\rho_{R R}^{0}\right),
\end{gathered}
$$




$$
\begin{gathered}
\rho_{j j}^{0}=\frac{e^{-\frac{E_{j}}{k T}}}{\sum_{i} e^{-\frac{E_{i}}{k T}}}, \quad \rho_{j \neq j}^{0}=0, \\
i \hbar \frac{\partial \rho_{L R}}{\partial t}=\left(\Delta-\frac{i \hbar}{\tau_{L R}}\right) \rho_{L R}-U\left(\rho_{L L}-\rho_{R R}\right), \quad \rho_{L R}=\rho_{R L}^{*},
\end{gathered}
$$

Диагональный матричный элемент $\rho_{L L}$ имеет смысл вероятности найти атом в левой яме. Исследуемая система для простоты характеризуется двумя временами релаксации: временем продольной релаксации системы $\tau_{L L}=\tau_{R R}$ с атомом в левой (правой) потенциальной яме и $\tau_{L R}=\tau_{R L}$ - временем поперечной релаксации недиагональных матричных элементов. Если период квантовых осцилляций много меньше времени продольной и поперечной релаксации, то процесс является когерентным; а вероятность туннельного перехода - осциллирующая функция времени, т.е. описывается квантовой кинетикой. Когда время туннельного перехода атома существенно превышает время продольной или поперечной релаксации, когерентность нарушается, и вероятность найти частицу в определенном состоянии может быть получена из решения классических кинетических уравнений.

Оценим температуру $T_{*}$, при которой частота квантового подбарьерного туннелирования атома $\omega_{K}$ становится равной частоте надбарьерного флуктуационного перескока $\omega_{T}$. Воспользовавшись формулой Гамова и законом Аррениуса, приравнивая показатели экспонент, получим

$$
\begin{aligned}
\omega_{K}=\omega_{0} e^{-\frac{a}{a_{d B}}}, & \omega_{T}=\omega_{0} e^{-\frac{V}{k T}}, \\
a_{d B}=\frac{\hbar}{\sqrt{2 m V}}, & T_{*}=\sqrt{\frac{V}{2 m}} \frac{\hbar}{a k},
\end{aligned}
$$

где $a_{d B}$ - длина волны де Бройля, $V, a$ - высота и ширина потенциального барьера в двухъямном потенциале. Из (8) видно, что $T_{*}$ с ростом $V$ увеличивается пропорционально $\sqrt{V}$ и с ростом $m$, а уменьшается пропорционально $\frac{1}{\sqrt{m}}, \frac{1}{a}$.

Для двухуровневой системы с жестким потенциалом характерные значения $V \approx 1 \mathrm{eV}, m \approx 10^{-25} \mathrm{~kg}$, $a_{d B} \approx 0.56 \cdot 10^{-12} \mathrm{~m}$. При ширине барьера $a=3 \cdot 10^{-11}$; $1.5 \cdot 10^{-11} ; 0.9 \cdot 10^{-11} \mathrm{~m}(0.1 ; 0.05 ; 0.03$ межатомного расстояния) получим соответственно $T_{*} \approx 65 ; 216 ; 721 \mathrm{~K}$ и частоту квантового туннелирования $\omega_{K} \approx 10^{-10} ; 20$; $10^{5} \mathrm{~Hz}$. Для структурного превращения с локальными смещениями атомов, много меньшими межатомного расстояния, эффект туннелирования является существенным для комнатных температур, а для более низких температур - главным. Поэтому возможным физическим механизмом неупругой деформации при комнатных температурах является квантовое туннелирование некоторых атомов в двухъямном потенциале, или атомных групп. Способность аморфных сплавов к неупругой необратимой деформации, к структурной релаксации, к образованию нанокластеров и нанокристалов связана с коллективизированным металлическим характером межатомной связи, при которой гораздо легче могут быть осуществлены процессы коллективных атомных перемещений.

\section{2. Локализованные состояния электрона в потенциальной нанометровой яме и образование фазона}

Допустим, что электрон совершает движение в трехмерной потенциальной яме, сферически симметричной относительно силового центра. В этом случае потенциальная энергия зависит только от расстояния электрона до силового центра - $U(r)$. Пусть трехмерная потенциальная яма имеет вид: $U(r)=-U_{0}$ при $r<a$, и равна нулю при $r>a$. В сферической системе координат уравнение Шредингера для стационарных состояний имеет вид [27]:

$$
\frac{1}{r^{2}} \frac{d}{d r}\left(r^{2} \frac{d}{d r} \Psi\right)+\frac{2 m}{\hbar^{2}}(E-U(r)) \Psi=0 .
$$

Введем новую функцию $\Phi=r \Psi$. Тогда уравнение (9) легко привести к виду

$$
\frac{d^{2}}{d r^{2}} \Phi+\frac{2 m}{\hbar^{2}}(E-U(r)) \Phi=0 .
$$

Это уравнение математически тождественно уравнению Шредингера для одномерного случая [27]. Однако нужно учесть граничное условие, что при $r=0$ функция $\Phi$ должна обращаться в нуль, так как в противном случае волновая функция $\Psi=\frac{\Phi}{r}$ обращалась бы в бесконечность. Тогда в качестве решений уравнения (10), конечных при $r=0$ и обращающихся в нуль при $r \rightarrow \infty$, можно взять

$$
\begin{gathered}
\Phi=B \sin (k r) \text { при } \quad r \leq a, \\
\Phi=C \exp (-\alpha r) \quad \text { при } \quad r>a, \\
k=\sqrt{\frac{2 m\left(E+U_{0}\right)}{\hbar^{2}}}, \quad \alpha=\sqrt{-\frac{2 m E}{\hbar^{2}}}
\end{gathered}
$$

(так как мы рассматриваем частицу внутри потенциальной ямы, необходимо считать $E<0)$. Таким образом, задача свелась к задаче о движении электрона в одномерной потенциальной яме, поэтому и уровни энергии определяются так же. Различие состоит только в том, что теперь необходимо отбросить состояния с четными волновыми функциями и оставить лишь состояния с нечетными волновыми функциями.

Принципиальное отличие одномерной потенциальной ямы от трехмерной состоит в том, что для одномерной ямы всегда существует по крайней мере одно собственное значение энергии с четной волновой функцией. 
В случае сферически симметричной прямоугольной ямы этого может и не быть. Из соотношения $k^{2}+\alpha^{2}=\frac{2 m}{\hbar^{2}} U_{0}$ видно, что если

$$
\frac{2 m U_{0} a^{2}}{\hbar^{2}}<\left(\frac{\pi}{2}\right)^{2}, \quad \text { т.е. } \quad U_{0}<\frac{\pi^{2} \hbar^{2}}{8 m a^{2}},
$$

то кривая, заданная уравнением $\operatorname{tg}\left(\frac{k a}{2}\right)=-\frac{k}{\alpha}$, никогда не пересечется с окружностью, заданной уравнением $k^{2}+\alpha^{2}=\frac{2 m}{\hbar^{2}} U_{0}$. Это означает, что при выполнении условия (12) в потенциальной яме не появится ни одного уровня дискретного спектра энергии из-за того, что мощность ямы слишком мала.

Подставляя значения фундаментальных констант $\hbar \cong 1 \cdot 10^{-34} \mathrm{~J}$, масса электрона $m=9 \cdot 10^{-31} \mathrm{~kg}$, для радиусов первой, второй, третьей координационной сферы $a_{1}=0.35 \cdot 10^{-9} \mathrm{~m}, a_{2}=0.7 \cdot 10^{-9} \mathrm{~m}, a_{3}=1.05 \cdot 10^{-9} \mathrm{~m}$, получаем соответственно $U_{0}\left(a_{1}\right)=0.7 \mathrm{eV}, \quad U_{0}\left(a_{2}\right)=$ $=0.18 \mathrm{eV}, U_{0}\left(a_{3}\right)=0.08 \mathrm{eV}$. Из физических соображений следует, что совершенный локальный порядок может распространяться, начиная с первой координационной сферы, и минимальный радиус потенциальной ямы равен межатомному расстоянию $a_{1}=0.35 \cdot 10^{-9} \mathrm{~m}$. Для условия возникновения локализованного состояния электрона в потенциальной яме с минимальным радиусом глубина потенциальной ямы возникшего нанокристалла должна быть приблизительно $1 \mathrm{eV}$.

Основным условием возникновения флуктуонов [16] является подвижность атомной системы, обеспечивающая возможность ее быстрой перестройки и образования флуктуаций при небольшом изменении термодинамического потенциала. Такая ситуация реализуется вблизи точки фазового перехода первого рода из аморфного в кристаллическое состояние, где плотности термодинамических потенциалов обеих структур становятся близки. При большой межфазной поверхностной энергии, присущей твердофазному превращению, флуктуационное образование области кристаллической фазы приводит к значительному изменению термодинамического потенциала и может оказаться нестабильным (неравновесным). Однако при наличии притяжения электрона проводимости к образовавшейся области кристаллической фазы электрон может в ней локализоваться. Если происходящее при этом понижение энергии электрона превосходит увеличение энергии термодинамического потенциала при образовании области кристаллической фазы, то флуктуация стабилизируется. Возникающее термодинамически устойчивое образование области новой фазы с локализованным в ней электроном (неравновесное в отсутствие электрона) называется фазоном [16]. Как показано в разд. 1, образование нанообласти кристаллической фазы может происходить также с помощью механизма квантового туннелирования, стимулированного неупругой деформацией, поэтому при таких условиях в нанообласти кристаллической фазы также возможна локализация электрона проводимости и образование фазона.
В простейшем случае фазон можно рассматривать как сферическую наночастицу кристаллической фазы радиуса $R$, которой соответствует потенциальная яма глубиной $-U$ относительно средней потенциальной энергии аморфного состояния. Изменение термодинамического потенциала $\Delta \Phi$ выражается формулой

$$
\begin{gathered}
\Delta \Phi(R)=\frac{4}{3} \pi R^{3} \varphi^{\prime}+4 \pi R^{2} \sigma_{s}+E_{e}(R), \\
E_{e}(R)=-U+\frac{\hbar^{2} k^{2}}{2 m_{*}},
\end{gathered}
$$

где $E_{e}(R)$ - энергия электрона в потенциальной яме глубиной $-U, m_{*}-$ эффективная масса электрона. Здесь $\varphi^{\prime}<0-$ разность плотностей термодинамических потенциалов кристаллического и аморфного состояний, $\sigma_{s}-$ межфазная поверхностная энергия. Первое слагаемое в (13) учитывает убывание потенциала $\Phi$ аморфного состояния при образовании фазона, второе - увеличение $\Phi$ за счет образования границы раздела фаз с дополнительной поверхностной энергией $\sigma_{s}$, третье описывает отрицательный вклад в $\Delta \Phi$ энергии локализованного электрона проводимости.

Убедимся, что $\Delta \Phi$ становится отрицательным (и фазоны стабильны) при $U$, больших некоторого значения $U_{0}$. Минимальному значению $\Delta \Phi$ соответствует критическое значение $R_{0}$, определяющее радиус стабильно существующего фазона. Определим значение $R_{0}$. Учтем, что стабильно существующему фазону соответствует связанное состояние электрона $E_{e}(R)<0$. Кинетическую энергию основного состояния электрона в потенциальной яме радиуса $R$ можно оценить из условия $2 R=\lambda=\frac{2 \pi}{k}$, где $\lambda-$ длина волны электрона. Подставляя $k$ в выражение для кинетической энергии электрона, находим

$$
\frac{\hbar^{2} k^{2}}{2 m_{*}}=\frac{\hbar^{2} \pi^{2}}{2 m_{*} R^{2}} .
$$

Тогда формула (13) приобретает вид

$$
\Delta \Phi(R)=\frac{4}{3} \pi R^{2} \varphi^{\prime}+4 \pi R^{2} \sigma_{s}-U+\frac{\hbar^{2} \pi^{2}}{2 m_{*} R^{2}} .
$$

Приравнивая $\partial \Delta \Phi(R) / \partial R=0$, находим $R_{0}^{4}\left[4 \pi \varphi^{\prime} m_{*} R_{0}+\right.$ $\left.+8 \pi \sigma_{s} m_{*}\right]=\hbar^{2} \pi^{2}$. При большой межфазной поверхностной энергии $4 \pi\left|\varphi^{\prime}\right| m_{*} R_{0} \ll 8 \pi \sigma_{s} m_{*}$ или $\left|\varphi^{\prime}\right| R_{0} \ll 2 \sigma_{s}$ получим

$$
R_{0}=\left(\frac{\hbar^{2} \pi}{8 \sigma_{s} m_{*}}\right)^{1 / 4}
$$

Радиус фазона (15) определяется величиной межфазной поверхностной энергии $\sigma_{s}$, причем возрастание $\sigma_{s}$ приводит к уменьшению радиуса фазона. Для значений $\sigma_{s}=20 \mathrm{erg} / \mathrm{cm}^{2}[16]$ и $\left|\varphi^{\prime}\right| R_{0} \ll 2 \sigma_{s}$ критический радиус фазона составляет $R_{0} \approx 0.5 \mathrm{~nm}$. Из физических соображений ясно, что минимальный радиус фазона равен межатомному расстоянию $a_{1}=0.35 \cdot 10^{-9} \mathrm{~m}$, поэтому значение критического радиуса фазона $R_{0} \approx 0.5 \mathrm{~nm}$ вполне разумно. 
Рассмотрим теперь условия образования фазона. Фазон образуется при таких значениях параметров системы, при которых величина $\Delta \Phi(R)$ становится отрицательной, т.е. при $\Delta \Phi\left(R_{0}\right)=0$. При выполнении условия $\varphi^{\prime} R_{0} \ll 2 \sigma_{s}$ из (14), пренебрегая первым слагаемым в правой части, получаем

$$
4 \pi R_{0}^{2} \sigma_{s}-U_{0}+\frac{\hbar^{2} \pi^{2}}{2 m_{*} R_{0}^{2}}=0 .
$$

Соотношение (16) определяет критическое значение $U_{0}$ глубины потенциальной ямы, при которой образуется фазон. Используя (16), (15), получаем

$$
U_{0}=8 \pi \sigma_{s} R_{0}^{2}=(2 \pi)^{3 / 2} \sqrt{\frac{\hbar^{2} \sigma_{s}}{m_{*}}} .
$$

При приведенных выше значениях $\sigma_{s}$ и $\varphi^{\prime} R_{0} \ll 2 \sigma_{s}$ величина $U_{0}$ составляет приблизительно $1 \mathrm{eV}$. Поскольку оценки радиуса фазона совпадают по порядку величины с экспериментально обнаруженными значениями радиуса нанокристалла [2-5], разумно предположить, что механизм возникновения нанокристалла в металлическом стекле - это механизм образования фазона.

\section{3. Модель неравновесного перехода аморфная структура - нанокристаллическая структура}

Отсутствие дальнего порядка во взаимном расположении атомов является определяющим признаком аморфных тел [29], поэтому описание их сложной структуры с помощью представлений только о параметре дальнего порядка, как для кристаллов, недостаточно. Топологическим называется порядок положений атомов тела безотносительно к типам последних. Локальный порядок атома определяется конфигурацией его первой координационной сферы, т. е. координационного многогранника. Экспериментально установлено, что в металлических стеклах, обладающих сильным топологическим беспорядком, часто реализуется довольно совершенный локальный порядок, с точностью до упругих искажений, совпадающий с локальным порядком в стабильных или метастабильных кристаллических телах того же состава $[2,3,30]$.

Полученная в работах $[2,3,30]$ структура аморфных тел хорошо описывается конгломератной моделью [31]. В этой модели структура твердого аморфного тела состоит из кластеров атомов, имеющих определенное ближнее упорядочение, которые погружены в среду с менее совершенным упорядочением, например в систему шаров со случайной плотной упаковкой. Дальнейшим развитием конгломератной модели стала концепция о поликластере - твердом теле, состоящим из локально регулярных кластеров (ЛРК), представляющих собой совокупности атомов, обладающих совершенным локальным порядком [24]. Существенной особенностью поликластера является то, что кластерные границы двумерны и имеют толщину монослоя. Релаксирующие к поликластеру неустойчивые аморфные структуры имеют области с несовершенным локальным порядком, которые не двумерны, а трехмерны. То есть ЛРК разделены трехмерными областями локального беспорядка. Структура межкластерных областей такова, что атомы в них не обладают каким либо из совершенных типов локального порядка, обеспечивающих максимальные значения энергии связи. Именно это обстоятельство позволяет заключить, что удельная свободная энергия в межкластерных областях выше, чем в локально регулярных кластерах. Благодаря этому релаксация аморфных структур начинается с перестройки межкластерных областей, в процессе которой атомы обретают локальное упорядочение. При этом возможно как достраивание регулярных продолжений существующих ЛРК, так и образование новых ЛРК.

Последний вариант релаксации энергетически выгоден в том случае, когда выигрыш в удельной свободной энергии вновь образуемого кластера превосходит энергию образования новых межкластерных границ. Если предположить, что энергия упругих деформаций в кластере растет пропорционально объему, то при достижении некоторого критического размера дальнейший рост ЛРК становится менее выгодным, чем образование границы, сопровождающееся релаксацией напряжений. В этом случае при релаксации аморфное тело приобретает нанокластерную структуру, если исходная структура не слишком сильно отличается от нанокластерной.

Для описания процесса релаксации аморфной (конгломератной) структуры в нанокластерную структуру введем безразмерную величину, характеризующую структурное состояние среды, которая называется параметром порядка $\eta$. Физически параметр порядка $\eta$ представляет собой объемную долю атомов, находящихся в ЛРК с совершенным локальным порядком определенного типа, в единице объема. В полностью аморфном состоянии параметр порядка $\eta$ равен нулю, а в нанокластерной структуре параметр порядка $\eta$ равен единице. Процесс структурной релаксации рассматривается как временная последовательность структурных состояний среды, описывающаяся изменением параметра порядка со временем, т. е. $\eta=\eta(t)$. Мы предполагаем, что по параметру $\eta$ аморфное состояние является неустойчивым, т. е. оно не отделено от локально устойчивого нанокластерного состояния энергетическим барьером. Структурная релаксация деформируемой неравновесной однородной системы к нанокластерной структуре описывается уравнением Ландау-Халатникова [32] для параметра порядка

$$
\begin{gathered}
\frac{\partial \eta}{\partial t}=-\alpha_{\eta}(T, A)(\eta-1), \\
\alpha_{\eta}(T, A)=\omega_{0}\left[e^{-\frac{\left(E_{\alpha}-\sigma \nu\right)}{k T}}+\Theta\left(A-A_{c}\right) e^{-\frac{a}{a_{d B}}}\right],
\end{gathered}
$$

где $\alpha_{\eta}(T, A)$ - скорость релаксации, которая содержит два члена различной физической природы. Первый, „тер- 
мический“, связан с локальной тепловой флуктуацией среды, а второй, „атермический“, - с туннелированием атома или группы атомов в двухъямном потенциале, стимулированным локальной неупругой деформацией среды. $E_{\alpha}$ - энергия активации атомной перестройки, $\sigma$ - механическое напряжение, $v$ - удельный объем на атом. $\Theta\left(A-A_{c}\right)$ - тэта-функция, которая отлична от нуля, когда амплитуда инфразвука $A$ вызывает неупругую необратимую деформацию. При циклической деформации среды параметр порядка возрастает пропорционально количеству циклов. При критическом значении числа циклов деформации $n_{c} \gg 1$, т. е. за время $t_{c}=n_{c} t_{i m p} \gg \alpha_{\eta}^{-1}$, среда полностью релаксирует в нанокластерную структуру.

Классический фазовый переход первого рода газ-жидкость протекает через образование критического зародыша новой фазы непосредственно в исходной фазе [32]. Однако образование нанокристаллов в объеме аморфной фазы значительно затрудняется дополнительными факторами. Например, плотность кристаллической фазы отличается от плотности аморфной структуры, поэтому для образования нанокристалла необходимо учесть упругие напряжения, возникающие как в нанокристалле, так и в аморфной матрице. Еще одним важным фактором, препятствующим образованию нанокристалла, являются упругие напряжения, возникающие изза когерентного сопряжения решеток кристаллической и аморфной структуры. Кроме того, при комнатной температуре низкая подвижность атомов в аморфном состоянии также тормозит образование нанокристаллов.

Итак, упругая энергия и другие факторы существенно увеличивают энергию образования нанокристаллов непосредственно в аморфной структуре, и это неоднородное состояние перестает играть роль активированного комплекса для структурного перехода. Нанокристаллы перестают соответствовать наинизшему энергетическому барьеру, разделяющему аморфную и кристаллическую структуры. Поэтому в процессе релаксации, стимулированной циклической деформацией, вначале из аморфного состояния образуется другое однородное состояние - нанокластерная структура, которое будет играть роль предпереходного состояния, а из него уже будут образовываться нанокристаллы.

Для описания процесса неравновесного перехода первого рода из аморфной структуры в нанокристаллическую введем еще одну безразмерную величину, характеризующую структурное состояние среды, которая называется параметром порядка $\varphi$. В случае наноструктурирования параметром порядка выбираем нормированную объемную долю атомов (нормированную на объемную долю насыщения), находящихся в нанокристаллах с совершенным локальным кристаллическим порядком, в единице объема. В аморфном состоянии параметр порядка равен нулю, а в нанокристаллическом состоянии параметр порядка равен единице. Процесс структурного превращения рассматривается как временная последовательность структурных состояний среды, описываю- щаяся изменением параметра порядка со временем, т.е. $\varphi=\varphi(t)$. Мы предполагаем, что по параметру $\varphi$ аморфное состояние является локально устойчивым, и оно отделено от расположенного ниже по свободной энергии локально устойчивого нанокристаллического состояния энергетическим барьером. При циклической деформации вначале происходит релаксация аморфной структуры в нанокластерную по параметру порядка $\eta$, при этом накапливается потенциальная энергия локальных статических смещений атомов (внутренняя энергия неупругой деформации среды). Поэтому энергетический барьер, разделяющий аморфное и нанокристаллическое состояния по параметру $\varphi$, уменьшается. При критическом значении числа циклов деформации $n_{c} \gg 1$ среда переходит в нанокластерное состояние, при котором этот энергетический барьер становится очень малым или даже равным нулю. Среда становится неустойчивой относительно образования нанокристаллов. Структурная релаксация деформируемой неравновесной аморфной системы к наноструктурному состоянию описывается уравнением Ландау-Халатникова [32] для параметра порядка $\varphi$

$$
\begin{gathered}
\frac{\partial \varphi}{\partial t}=-\alpha_{\varphi}(T, A) \varphi\left(\varphi-\frac{1}{2}[1-\eta]\right)(\varphi-1), \\
\alpha_{\varphi}(T, A)=\omega_{0}\left[e^{-\frac{\left(E_{\beta}-E_{e}-\sigma v\right)}{k T}}+\Theta\left(A-A_{c}\right) e^{-\frac{a}{a_{d B}}}\right],
\end{gathered}
$$

где $\alpha_{\varphi}(T, A)-$ скорость релаксации, которая содержит два члена различной физической природы. Первый, „термический“, связан с локальной тепловой флуктуацией среды, а второй, „,атермический“, - с туннелированием группы атомов в двухъямном потенциале, стимулированным локальной неупругой деформацией среды. $E_{\beta}$ - энергия активации возникновения нанокристалла, $E_{e}$ - энергия локализованного электрона проводимости в локальной потенциальной яме нанокристалла. При циклической деформации среды параметр порядка $\eta$ возрастает пропорционально количеству циклов. При критическом значении числа циклов деформации $n_{c} \gg 1$, т.е. за время $t_{c}=n_{c} t_{i m p}$, среда полностью релаксирует в нанокластерную структуру $\eta \rightarrow 1$, а энергетический барьер по параметру $\varphi$ обращается в нуль и начинается образование нанокристаллов.

Так как процесс образования нанокристаллов непосредственно в аморфной структуре подавлен, неравновесный переход первого рода вынужден протекать через предпереходное состояние - нанокластерную структуру и описывается двумя параметрами $(\eta, \varphi)$, подчиняющимися уравнениям (18), (20). Из нанокластерного состояния система попадает в наноструктурное состояние через наивысшую точку наинизшего энергетического барьера в пространстве координат фазового перехода $(\eta, \varphi)$, т. е. седловую точку.

Построим модельный потенциал данного фазового перехода $F(\eta, \varphi)$ в пространстве координат фазового 
перехода $(\eta, \varphi)$. Проведенный анализ (18), (20) показывает, что переход с двумя координатами $(\eta, \varphi)$ может быть смоделирован следующей зависимостью модельного потенциала

$$
\begin{gathered}
F(\eta, \varphi)=\frac{1}{2}[\eta-1]^{2}+A(\eta) \varphi^{2}+B(\eta) \varphi^{3}+\frac{\varphi^{4}}{4}, \\
A(\eta)=\frac{1}{4}[1+\eta], \quad B(\eta)=-\frac{1}{2}\left[1+\frac{\eta}{3}\right] .
\end{gathered}
$$

При $\eta=0$ коэффициенты $A(0)=\frac{1}{4}, B(0)=-\frac{1}{2}$, а простейший потенциал $F(0, \varphi)$ - двухъямный и симметричный.

При фиксированном параметре $0 \leq \eta<1$ функция $F(\eta, \varphi)$ имеет два минимума при $\varphi_{1}=0, \varphi_{3}=1$ и один максимум при $\varphi_{2}=\frac{1}{2}[1-\eta]$. Причем для

$$
\begin{gathered}
\Delta F(\eta, \varphi)=F(\eta, \varphi)-\frac{1}{2}[\eta-1]^{2}, \quad \Delta F\left(\eta, \varphi_{1}\right)=0, \\
\Delta F\left(\eta, \varphi_{2}\right)=\frac{1}{64}[1-\eta]^{3}\left[1+\frac{\eta}{3}\right], \Delta F\left(\eta, \varphi_{3}\right)=-\frac{\eta}{12} .
\end{gathered}
$$

Таким образом, при фиксированном параметре $0 \leq \eta<1$ функция $F(\eta, \varphi)$ может описать неравновесный переход первого рода аморфное $\left(\varphi_{1}=0\right)$-нанокристаллическое состояние $\left(\varphi_{3}=1\right)$ через потенциальный барьер $\Delta F\left(\eta, \varphi_{2}(\eta)\right)$. Анализируя (22), получим, что функция $F(\eta, \varphi)$ в области определения переменных $0 \leq \eta<1,0 \leq \varphi \leq 1$ имеет два минимума $(\eta, 0),(\eta, 1)$; один максимум $\left(0, \frac{1}{2}\right)$ и три седловые точки $(0,0)$, $(0,1), \quad\left(\eta, \frac{1}{2}[1-\eta]\right)$. При $\eta=1$ потенциальный барьер $\Delta F\left(\eta, \varphi_{2}(\eta)\right)$ исчезает, точка $(1,0)$ становится неустойчивой седловой, остается только один минимум $(1,1)$.

Из начального состояния $(0,0)$ можно сразу попасть в конечное $(1,1)$, используя, например, стехиометрическое соотношение $\eta=\varphi$. Но при этом придется преодолевать довольно большой барьер (почти равный максимуму), так как $\Delta F\left(\eta, \varphi_{2}\right)$ может быть достаточно большим. Это классический механизм фазового перехода первого рода.

При превращении аморфное состояние-наноструктура барьер перехода является слишком большим из-за упругой энергии образования нанокристалла. Но есть другой путь - через предпереходное состояние $(\eta \rightarrow 1,0)$. В этом состоянии $\varphi_{2}=\frac{1}{2}[1-\eta]$ стремится к нулю, поэтому барьер, который нужно преодолеть, чтобы попасть в наноструктурное состояние, также стремится к нулю $\Delta F\left(\eta, \varphi_{2}\right) \rightarrow 0$. Значительно выгоднее вначале осуществить переход вдоль координаты $\eta$ из 0 в 1, что приводит к резкому уменьшению барьера $\Delta F\left(\eta, \varphi_{2}\right)$ вдоль координаты $\varphi$. И лишь затем выполнить переход вдоль координаты $\varphi$ из 0 в 1.

\section{4. Оценка модельных параметров и выводы}

Проведем оценку величины плотности запасенной потенциальной энергии статических смещений атомов (неупругой деформации) при инфразвуковом воздействии на аморфную пленку. Из эксперимента $[4,5]$ известны размеры пленки: длина $l=1 \cdot 10^{-2} \mathrm{~m}$, $r=1.6 \cdot 10^{-3} \mathrm{~m}$, толщина $d=4 \cdot 10^{-5} \mathrm{~m}$. Время циклического механического воздействия $10 \mathrm{~min}$, частота воздействия $20 \mathrm{~Hz}$. Амплитуда растягивающего смещения инфразвука равна $A=4 \cdot 10^{-6} \mathrm{~m}$, относительное продольное удлинение пленки составляет $\varepsilon=\frac{A}{l}=4 \cdot 10^{-4}$. Известно, что плотность потенциальной энергии однородной продольной упругой деформации равна $e=\frac{E}{2} \varepsilon^{2}$, где $E$ - модуль продольной упругости (модуль Юнга). Аморфная пленка является сплавом, в котором по составу основными элементами являются никель (45 процентов) и титан (41 процент). Известно, что $E_{\mathrm{Ni}} \approx 220 \mathrm{GPa}, E_{\mathrm{Ti}} \approx 110 \mathrm{GPa}$, среднее значение которых равно $E \approx 165 \mathrm{GPa}$. С учетом среднего значения $E \approx 165 \mathrm{GPa}$ и относительного удлинения $\varepsilon=\frac{A}{l}=4 \cdot 10^{-4}$ плотность потенциальной энергии равна $e \approx 1.3 \cdot 10^{4} \mathrm{~J} / \mathrm{m}^{3}$.

Для наглядности вычислим потенциальную энергию однородной продольной упругой деформации на один атом среды. Поскольку концентрация атомов в среде приблизительно равна $0.9 \cdot 10^{29}$ atom $/ \mathrm{m}^{3}$, приходящаяся на один атом энергия за один цикл деформации равна $e_{a} \approx 1 \cdot 10^{-6} \mathrm{eV} /$ atom. За время воздействия инфразвука происходит $n=12000$ циклов механической деформации, поэтому энергия, поступившая в систему за все время воздействия, составляет $n e_{a} \approx 1.2 \cdot 10^{-2} \mathrm{eV} /$ atom. Очевидно, что только малая часть (например, 10 процентов) этой энергии может превращаться в потенциальную энергию неупругой деформации. Поэтому величина запасенной потенциальной энергии неупругой деформации составляет 0.1 ne $a$ $\approx 1.2 \cdot 10^{-3} \mathrm{eV} /$ atom, а это меньше, чем кинетическая энергия атома при комнатной температуре $k T_{0} \approx 0.024 \mathrm{eV} /$ atom.

Известно, что скрытая теплота аморфно-кристаллического превращения, определяемая через разность (перепад) температур между аморфным и кристаллическим состояниями среды $\Delta T \approx 150 \mathrm{~K}$ [33], составляет $k \Delta T \approx 0.012 \mathrm{eV} /$ atom. Экспериментально установлено [4], что за 12000 циклов инфразвукового воздействия теплота аморфно-кристаллического превращения немного уменьшается. Поэтому свободная энергия аморфного состояния с нанокристаллами немного понижается по сравнению со свободной энергией аморфного состояния. Разумно предположить, что скрытая теплота превращения аморфного состояния в наноструктурное состояние много меньше, чем $k \Delta T \approx 0.012 \mathrm{eV} /$ atom, например $0.1 k \Delta T \approx 0.0012 \mathrm{eV} /$ atom. Таким образом, скрытая теплота превращения аморфного состояния в на- 
ноструктурное состояние $0.1 k \Delta T \approx 0.0012 \mathrm{eV} /$ atom по порядку величины совпадает с величиной запасенной потенциальной энергии неупругой деформации $0.1 n e_{a} \approx 1.2 \cdot 10^{-3} \mathrm{eV} /$ atom.

Поэтому можно сделать вывод, что физическими причиной и условием возникновения неустойчивости аморфного состояния относительно перехода в наноструктурное состояние (образования нанокристаллов) является накопление потенциальной энергии неупругой деформации до критического значения, равного скрытой теплоте превращения аморфного состояния в наноструктурное состояние.

Проведенная оценка величины плотности запасенной потенциальной энергии неупругой деформации, полученные физические причина и условие возникновения неустойчивости аморфного состояния позволяют сформулировать следующую физическую картину неравновесного перехода в наноструктурное состояние, а также возможные микроскопические механизмы релаксации в нанокластерное состояние и возникновения нанокристаллов.

Мы предполагаем, что при слабом воздействии инфразвука с амплитудой $1 \mu \mathrm{m}$ среда испытывает только динамические смещения атомов (колебания атомов около положений равновесия), релаксация среды идет медленно за счет тепловых флуктуаций, аморфная структура изменяется слабо. Внешняя механическая работа превращается в упругую энергию колебаний среды, а затем эта упругая энергия колебаний (за счет фонон-фононных взаимодействий, рассеяния фононов на неупорядоченной решетке и т. д.) превращается в тепловую энергию среды.

При сильном воздействии инфразвука с амплитудой $4 \mu \mathrm{m}$ наряду с динамическими смещениями атомов среда испытывает статические смещения атомов в новые положения равновесия (неупругую деформацию), происходит заметное увеличение скорости релаксации аморфной среды в нанокластерное состояние. Внешняя механическая работа превращается как в упругую энергию колебаний среды, так и запасается в виде потенциальной энергии статических смещений атомов (неупругой деформации). При каждом последующем цикле механической нагрузки величина неупругой деформации среды увеличивается, аморфная структура среды все более изменяется (среда быстро релаксирует в нанокластерное состояние). Соответственно запасенная потенциальная энергия неупругой деформации среды последовательно увеличивается. При критическом числе инфразвуковых колебаний запасенная потенциальная энергия неупругой деформации достигает критического значения (равного величине скрытой теплоты превращения), при котором энергетический барьер, разделяющий аморфное и нанокристаллическое состояние, исчезает. Аморфная среда становится абсолютно неустойчивой относительно образования нанокристаллов.

Физические механизмы релаксации среды в нанокластерное состояние и возникновения нанокристаллов, стимулированные инфразвуком при комнатной тем- пературе, следующие. В начальный момент времени среда представляет собой аморфную матрицу с распределенными в ней неупорядоченными нанообластями, содержащими свободный объем, и упорядоченными некристаллическими нанокластерами. При однократном воздействии инфразвука на среду атом на периферии нанокластера или нанообласти со свободным объемом, находящийся в двухъямном потенциале, или группа атомов, способных образовать две конфигурации с мало отличающимися энергиями, способны испытывать как тепловую флуктуацию (термический механизм), так и квантово туннелировать в новые положения (атермический механизм). Для структурного превращения с локальными статическими смещениями атомов, много меньшими межатомного расстояния, при комнатной температуре частота туннелирования больше, чем частота теплового перескока. Таким образом, при комнатной температуре и более низких температурах превалирует атермический (квантовый) механизм неупругой деформации. В результате неупругой деформации размер кластера с совершенным локальным порядком увеличивается, а из нанообласти со свободным объемом может возникнуть новый нанокластер с совершенным локальным порядком.

Поскольку времена релаксации и туннельного перехода по-разному зависят от температуры, оба механизма - термический и атермический — реализуются при деформировании металлических стекол. При понижении температуры тепловое движение атомов замораживается, что приводит к экспоненциальному уменьшению скорости релаксации по закону Аррениуса. Следовательно, при низких температурах скорость туннельного перехода превышает скорость релаксации и будет происходить когерентное туннелирование. При повышении температуры скорость релаксации возрастает быстрее скорости туннелирования. Эти величины вначале сравниваются, а затем скорость релаксации может существенно превысить скорость туннелирования. Процесс перехода становится термофлуктуационным. Таким образом, меняя температуру, можно менять кинетику процесса неупругой деформации металлических стекол от квантовой до классической.

При многократном воздействии инфразвука нанокластер растет и достигает такого критического размера, что его тепловая флуктуация - менее вероятный акт перестройки атомов в кристаллическое состояние или квантовое туннелирование - более вероятный акт перестройки атомов - создает локальную потенциальной яму, в которой возникает связанное состояние электрона проводимости. Если электрон притягивается к области нестабильной кристаллической фазы и локализуется в ней, то понижение энергии электрона может скомпенсировать поверхностную энергию межфазной границы и возникает стабильное образование фазон. Радиус фазона определяется величиной межфазной поверхностной энергией $\sigma_{s}$, причем возрастание $\sigma_{s}$ приводит к уменьшению радиуса фазона. Физически 
радиус фазона не может быть меньше радиуса первой координационной сферы. Для значений $\sigma_{s}=20 \mathrm{erg} / \mathrm{sm}^{2}$ критический радиус фазона равен $R_{0} \approx 0.5 \mathrm{~nm}$. Критическое значение глубины потенциальной ямы (относительно средней потенциальной энергии аморфного состояния), т.е. условие, при котором образуется фазон с $R_{0} \approx 0.5 \mathrm{~nm}$, составляет приблизительно $1 \mathrm{eV}$. Поскольку критический радиус фазона совпадает по порядку величины с экспериментально обнаруженным значением радиуса нанокристалла [2-5], разумно предположить, что механизм возникновения нанокристалла в металлическом стекле - это механизм образования фазона. Когда электроны проводимости локализуются на возникших нанокристаллах, они понижают энергию системы, в результате чего состояние с нанокристаллами становится устойчивым и возникает локально устойчивая наноструктура (по отношению к глобально устойчивой кристаллической структуре).

Экспериментально установлено [2-5], что морфология нанокристаллов зависит от способа нанокристаллизации. Различие в морфологии нанокристаллов, изотропной при отжиге $[2,3]$ и анизотропной при воздействии инфразвуком $[4,5]$ связано с разными механизмами зарождения и роста нанокристаллов. Во время нанокристаллизации высокотемпературным отжигом зарождение и рост нанокристаллов контролируются термофлуктуационными процессами. Однако при комнатной температуре вклад термофлуктуационных процессов мал, и неупругая деформация, структурная релаксация, нанокристаллизация протекает за счет квантовых переходов в наноразмерных двухуровневых системах, стимулированных инфразвуковыми колебаниями. Анизотропия формы нанокристалла определяется тензором потенциалов деформации, который зависит от локальной структуры стекла в том месте, где расположена двухуровневая система.

\section{Заключение}

Таким образом, предлагаемая модель кинетики позволяет сформулировать причину, условие, микроскопический механизм аморфно-наноструктурного превращения в металлических стеклах, инициированного воздействием инфразвука при комнатной температуре, количественно и качественно описать результаты и закономерности, полученные экспериментально.

\section{Финансирование работы}

Работа выполнена в рамках государственного задания ИФПМ СО РАН, тема номер FWRW-2019-0031.

\section{Конфликт интересов}

Авторы заявляют, что у них нет конфликта интересов.

\section{Список литературы}

[1] M.E. McHenry, M.A. Willard, D.E. Laughlin. Progress in Material Science, 44 (4), 291 (1999).

[2] К. Судзуки, Х. Худзимори, К. Хасимото. Аморбные металльь (Металлургия, М., 1987)

[3] А.М. Глезер, Н.А. Шурыгина. Аморфно-нанокристаллические сплавы (Физматлит, М., 2013)

[4] S. Belyaev, N. Resnina, V. Rubanik, A. Shelyakov, V. Niapomniashchay, E. Ubyivovk, I. Kasatkin. Materi. Lett., 209, 231 (2017).

[5] S. Belyaev, V. Rubanikjr, N. Resnina, V. Rubanik, E. Ubyivovk, E. Demidova, A. Uzhekina, I. Kasatkin, A. Shelyakov. Mater. Lett., 275, 128084 (2020).

[6] Я.И. Френкель. Введение в теорию металлов (Гос. изд-во технико-теорет. лит., М.-Л., 1950). Я.И. Френкель. Кинетическая теория жсидкостей (Наука, Л., 1975)

[7] W.K. Burton, N. Cabrera, F.C. Frank. Phil. Trans. Roy. Soc., 243, 299 (1951).

[8] U. Köster, U. Herold. Crystallization of Metallic Glasses in Topics in Applied Physics: Glassy Metals I, eds. H.-

J. Guntherrodt, H. Beck. (Springer-Verlad, Berlin, 1981), p. 225.

[9] A.L. Greer. Acta Metall., 30, 171 (1982).

[10] U. Köster, J. Meibhardt, Y. Birol, A. Aronin. Z. Metallkd, 86, 171 (1995).

[11] P.W. Anderson, B.I. Halperin, C.M. Varma. Philosophical Magazine, 25 (1), 1 (1972).

[12] М.И. Клингер. УФН, 152, 623 (1987).

[13] Е.Е. Слядников. ФТТ, 46 (6), 1065 (2004).

[14] Р. Пайерлс. Квантовая теория твердых тел (ИИЛ, М., 1956)

[15] Н. Мотт, Э. Дэвис. Электронные процессы в некристаллических веществах (Мир, М., 1982)

[16] М.А. Кривоглаз. УФН, 111 (4), 617 (1973).

[17] A.S. Argon. Acta Metall., 27, 47 (1979).

[18] A.I. Taub, F. Spaepen. Acta Met., 28 (10), 1781 (1980).

[19] M.R.J. Gibbs, J.E. Evetts, J.A. Leake. J. Mater. Sci., 18 (1), 278 (1983).

[20] А.Т. Косилов, В.А. Хоник. Изв. РАН. Сер. физическая, 57 (11), 192 (1993).

[21] T. Egami, V. Vitek. J. Non-Cryst. Sol., 62 (4), 499 (1984).

[22] Д.В. Лузгин, В.И. Полькин. Известия вузов. Цветная металлургия, 6, 71 (2016).

[23] F. Spaepen. Defects in amorphous metals. Les. Houches Lectures XXXV on Physics of Defects, Amsterdam: North Holland Press, 1981, p. 133.

[24] А.С. Бакай. Поликластерные аморфные тела (Синтекс, Харьков, 2013)

[25] В.С. Горский. ЖТФ, 6 (3), 272 (1936).

[26] В.И. Архаров. Мезоскопические явления в твердых телах и их микроструктура, в кн. Проблемы современной бизики (Наука, М., 1980), с. 357, В.И. Архаров, Изв. АН СССР, сер. физизическая, 28, 152 (1964).

[27] Л.Д. Ландау, И.М. Лифшиц. Квантовая механика (Наука, М., 1989)

[28] Р. Пантел, Г. Путхоф. Основы квантовой электроники (Мир, М., 1972) 
[29] Дж. Займан. Модели беспорядка (Мир, М., 1982)

[30] J. Hafner. Le Journal de Physique Colloques, 46, 9 (1985).

[31] А. Уббелоде. Плавление и кристаллическая структура (Мир, М., 1969)

[32] Л.Д. Ландау, Е.М. Лифшиц. Физическая кинетика (Наука, М., 2001)

[33] А.С. Рогачев, С.Г. Вадченко, А.С. Щукин, И.Д. Ковалев, А.С. Аронин. Письма в ЖЭТФ, 104 (10), 740 (2016). 